\title{
Automated Test System with Input Parameters Sweep-Function for Analog-to- Digital Converters
}

\author{
WeiWei Qin ${ }^{1,2}$, Sai-Weng Sin ${ }^{1,2}$, Seng-Pan $U^{1,2,3}$, Rui Paulo Martins ${ }^{1,2,4}$ \\ ${ }^{1}$ State Key Laboratory of Analog and Mixed-Signal VLSI, University of Macau, Macau, China \\ ${ }^{2}$ Department of ECE, Faculty of Science and Technology, University of Macau, Macau, China \\ ${ }^{3}$ Synopsys Macau Ltd. \\ ${ }^{4}$ On leave from Instituto Superior Técnico/Universidade de Lisboa, Portugal \\ Email: terryssw@umac.mo
}

Keywords: Analog to Digital Converter, Test System, Automated, Sweep Function, LabVIEW.

\begin{abstract}
This paper describes an automated test system for Analog to Digital Converters (ADC) combining various instruments controlled by LabVIEW, to implement standardbased real-time measurement which accurately characterizes the statistical and dynamic performances. Moreover, the proposed system provides an automated input parameter sweep-function, which realizes the ADC intelligent automation test with different input settings. Virtual Instruments (VIs) were created in system program to control signal generation and data acquisition. The system is not limited to the type or speed of the ADC under test due to its high compatibility and hardware modularity. We describe in detail the hardware arrangement and software programming, as well as the experimental testing of real ADCs, to demonstrate system's performance.
\end{abstract}

\section{Introduction}

In mixed-signal system, ADC plays a crucial role and to ensure their performance, low cost and high efficient ADC test methodologies are necessary. Some significant works have been done in the past. The authors in [1] introduced an industrial test bench for ADC. The bench hardware has been composed with known devices of instrumentation, with the whole hardware handled by software written with LabVIEW development system. However, this test-bench is slow since the LabVIEW code execution is slow in the performance calculation. In [2], a linearity test was presented for testing ADC statistical parameters in LabVIEW, but the dynamic parameters test weren't included. Reference [3] described an automated test system for ADC, based on an arrangement of equipment controlled by LabVIEW to implement standard-based procedures that provide accurate values of the relevant metrics of an ADC, and a reliable ADC testing method using LabVIEW was presented in [4]. However, both of them do not have input parameter sweep-function, and it is difficult to let the user to quickly gather the comprehensive information of the ADC with different input parameters. 
This work, devoted to design an automated test system for parametric and functional control of various instruments, is able to characterize fully an ADC. The proposed system differs from previously reported solutions because it allows the user to sweep automatically the input parameters for any types of ADC testing. The remaining part of this paper is organized as follows: Section 2 presents a brief review of the ADC under test; Section 3 describes, in detail, the proposed method including the hardware arrangement and software programming. Section 4 provides the experimental results about the measurement using the proposed test system, while Section 5 draws the conclusions.

\section{Device Under Test}

The device under test (DUT) in this example in the paper is a 4-channel continuous-time (CT) and discrete-time (DT) hybrid sigma-delta ADC [5]. This ADC chip is fabricated with 65nm CMOS technology, including a gain programmability function and 4-phase clock generation, and occupies an active chip area of $0.03 \mathrm{~mm}^{2}$ per channel.

\section{The Proposed Test Method}

The automated test system for ADC, developed based on different measurement devices, allows ADCs to be tested following the methods described by IEEE standards 1241-2010[6]. From the workstation containing LabVIEW, all the necessary instruments can be controlled remotely for quick setup of parameters needed. All instruments use the GPIB connection for serial communication with the system, except for the logic analyzer, which uses a TCP/IP connection for data transfer and communication. The completed acquisition and control system are developed by using LabVIEW program, and LabVIEW VIs control the measurement system, which we divided into sub-VIs for various routines including analog signal generation, digital acquisition, input parameter sweep and so on.

\subsection{Hardware Arrangement}

The hardware arrangement of the proposed method is showed in Fig. 1. The testing signal and clock signal are originated from two precision signal generators, and the high-pass and low-pass filters are utilized to confine out-of-band noise to avoid influence in the testing and clock signals. Moreover, the tunable bandpass filters are used in the proposed system, which can be programmed to set the filter center frequency. Several groups of power supply are used for the ADC under test and the digital output code, collected by a logic analyzer, which plays the role of code grabber. 


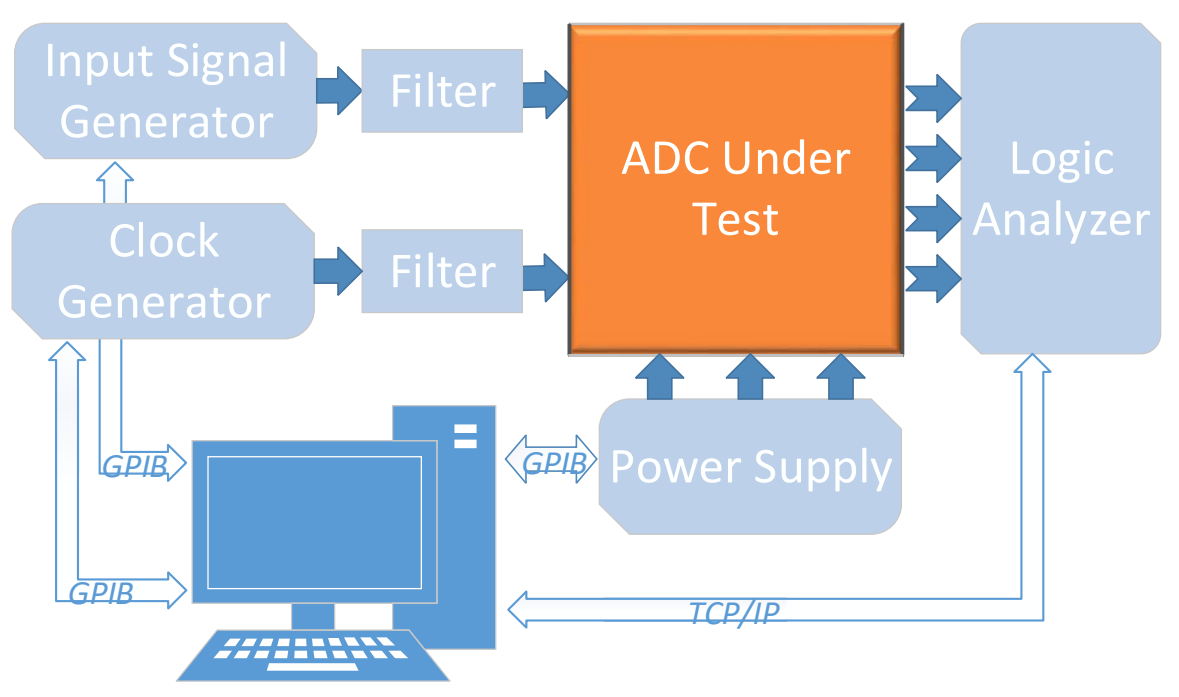

Figure 1 Hardware arrangement of the proposed automated test system.

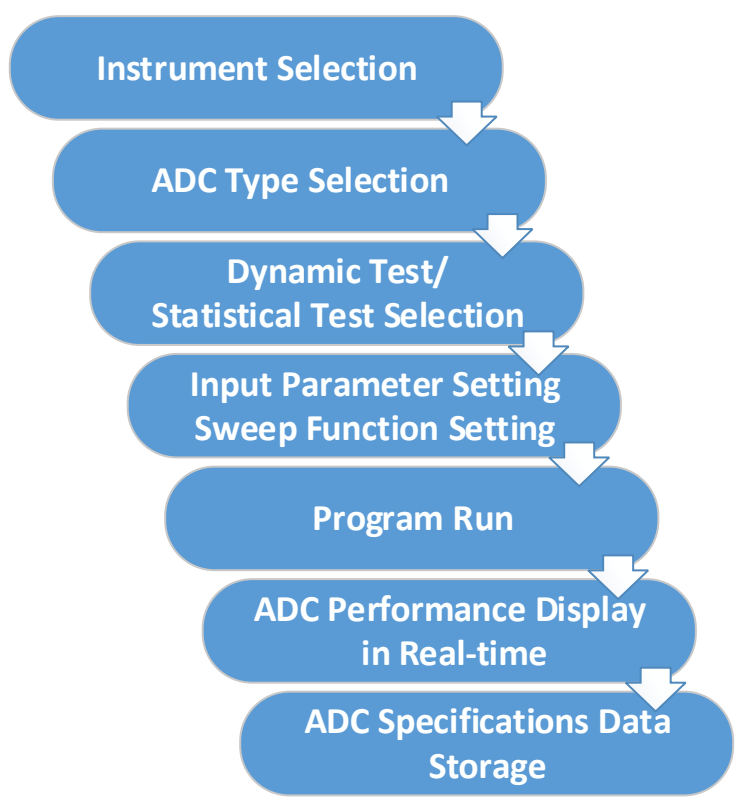

Figure 2 Test flow of the proposed test system. 


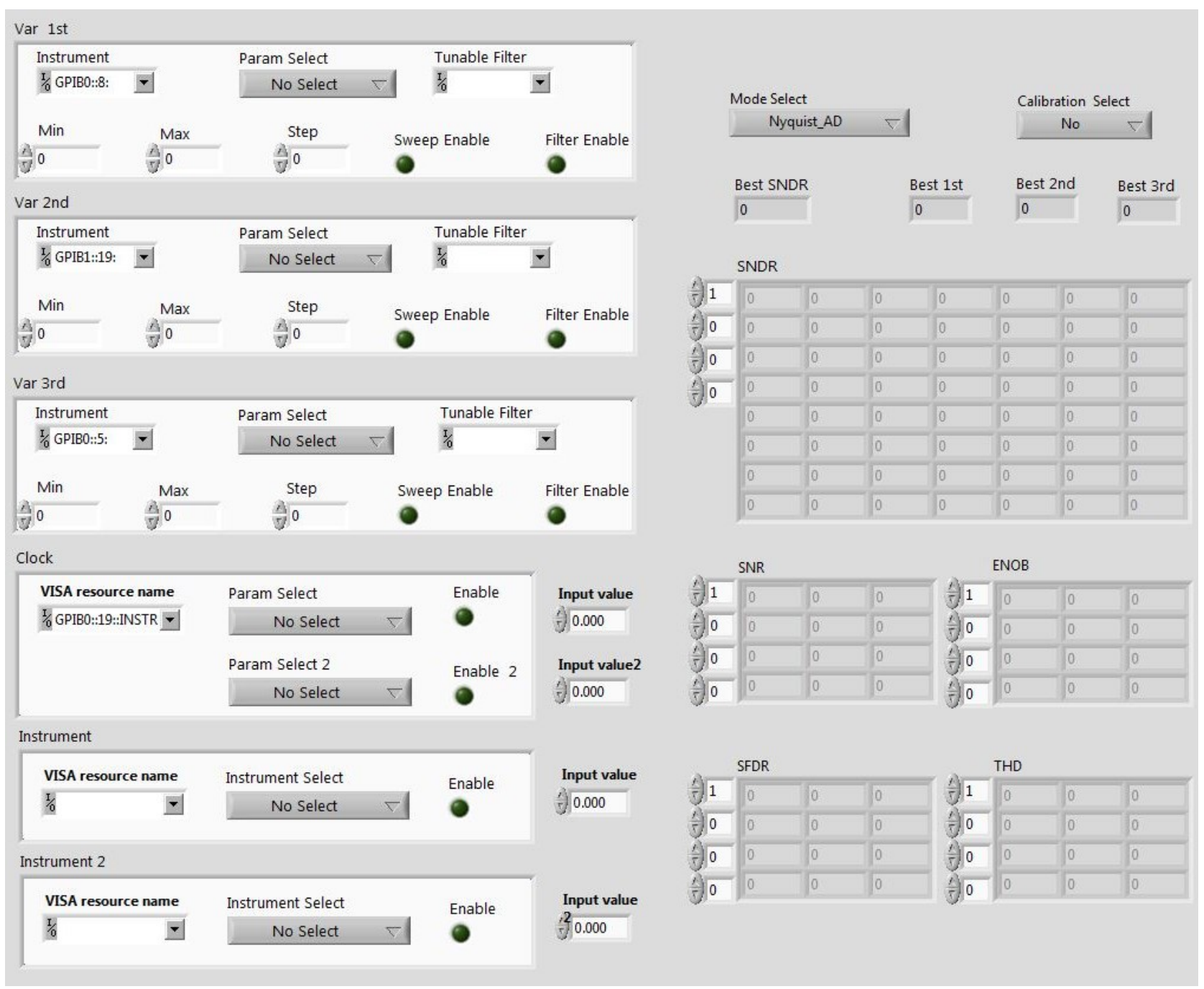

Figure 3 Top-level front panel of the test setup and performance tables.

\subsection{Software Programming}

The managing software was developed in LabVIEW. The ADC measurements are divided into two parts that almost cover all the parameters for the chip. One of the test parts is dynamic performance, and it includes Signal-to-noise and distortion ratio (SNDR), Signal-to-noise ratio (SNR), Total harmonic distortion (THD), and the Effective number of bits (ENOB). The other one is static performance, which includes the Integral nonlinearity (INL) and Differential nonlinearity (DNL). Histogram-based approach and spectral analysis (FFT) are applied in the proposed system [7]. In action, the system is executed following the diagram flow in Figure 2. The software program started by selecting the required instruments and making the measurement function setting about the ADC under test. Then all the instruments controlled by the program start running and the ADC begin to work. We apply the Matlab function to calculate the ADC specifications immediately, selected before and displayed in real-time once the output code is collected. In this way, it can help to save considerable time and make the test more efficient. All the specifications data are stored when all test runs are completed.

Fig. 3 shows the top-level front panel of the proposed automated test program, where the test results presented in the tables on the right detail every result accompanied by the corresponding input parameter index (also in the table). The input parameter sweep-function can be set in the tabs 
on the middle of this front panel. The requirements in terms of generators and input parameters (frequency of input signal, the amplitude of input signal, input power, etc.) can be selected and set. Once the program starts to run and the sweep-function configured as enable, the ADC test will start with the selected input parameters automatically changed according to the user setting. On the other hand, the program can also run with the specified input parameters without the sweep function. The program block diagram about some test setup and the input parameter sweep function is presented in Fig. 4. The For-Loop are employed to implement this functionality, and there are three loops in this routine since the proposed program can interleavely sweep three groups of input parameters in

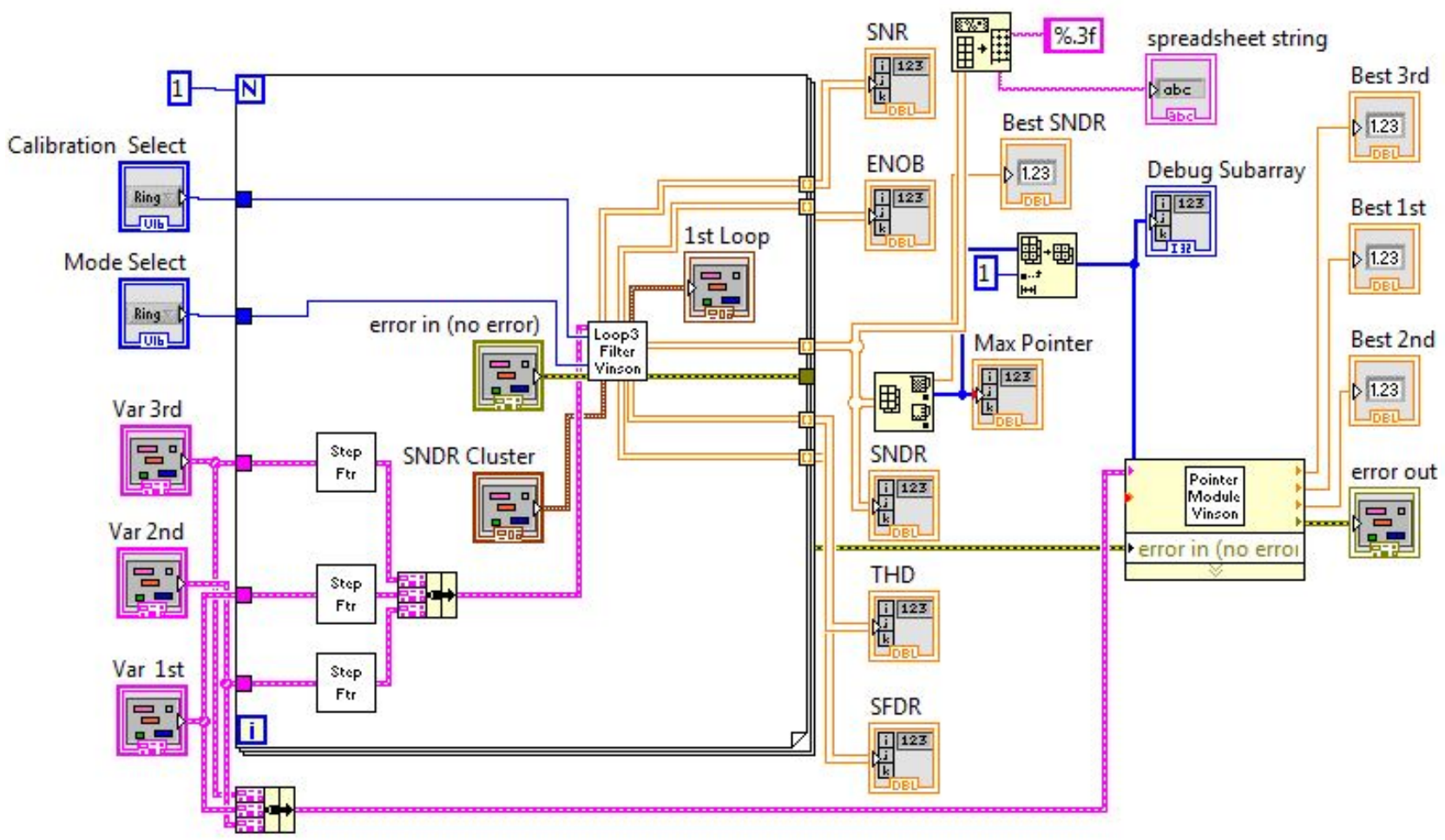

Figure 4 Software block diagram of the test setup and performance display.

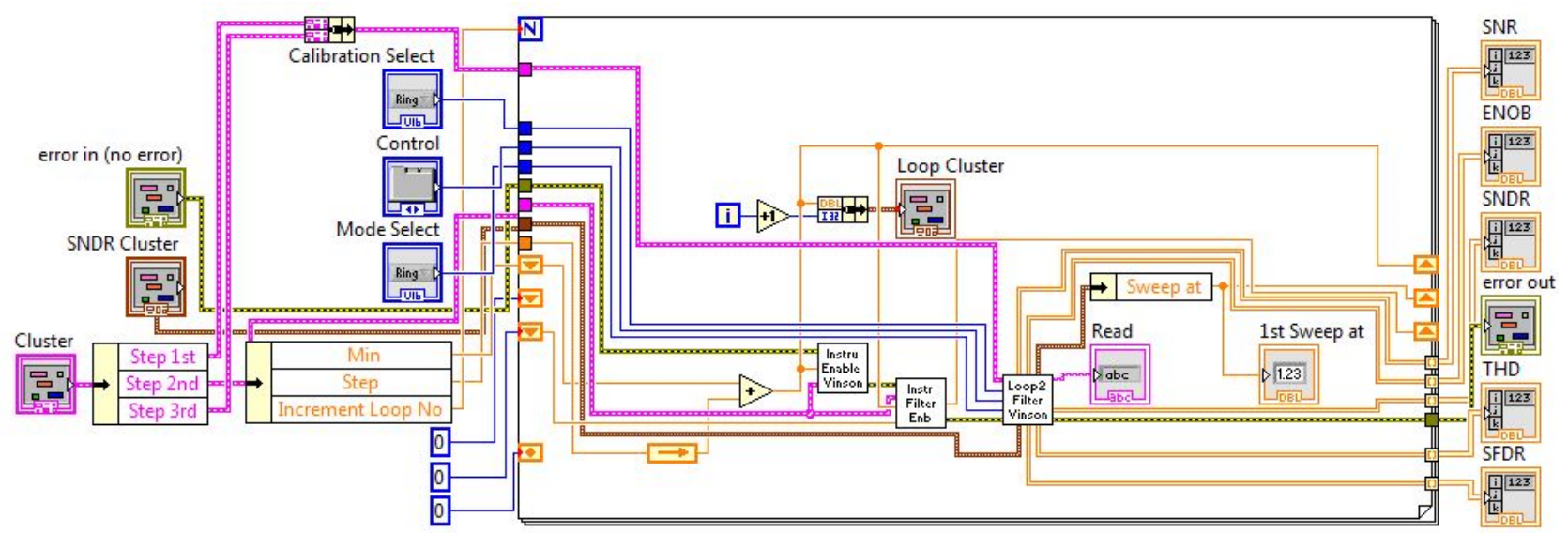

Figure 5 Software block diagram of one sweep loop.

one test suit. The details about one sweep loop are illustrated in Fig 5. The program allows the user to choose the instruments, maximum value, minimum value and step value in menus. The menus are built dynamically at the program front panel by reading "Var" tabs which contain the available 
instruments list and some other options. Fig. 6 illustrates the LabVIEW routine that performs the setting of the instrument parameters. The used Case-Structure has several cases which allow the user to set the different input parameters on different instruments, and this demonstrates the compatibility and flexibility of the proposed system. Also, some tabs in other sub-VIs allow the user to specify the number of samples, measurement options (statistical/dynamic), FFT windowing, plot options, the setup of logic analyzer, file selection for data $\log$ of the results.

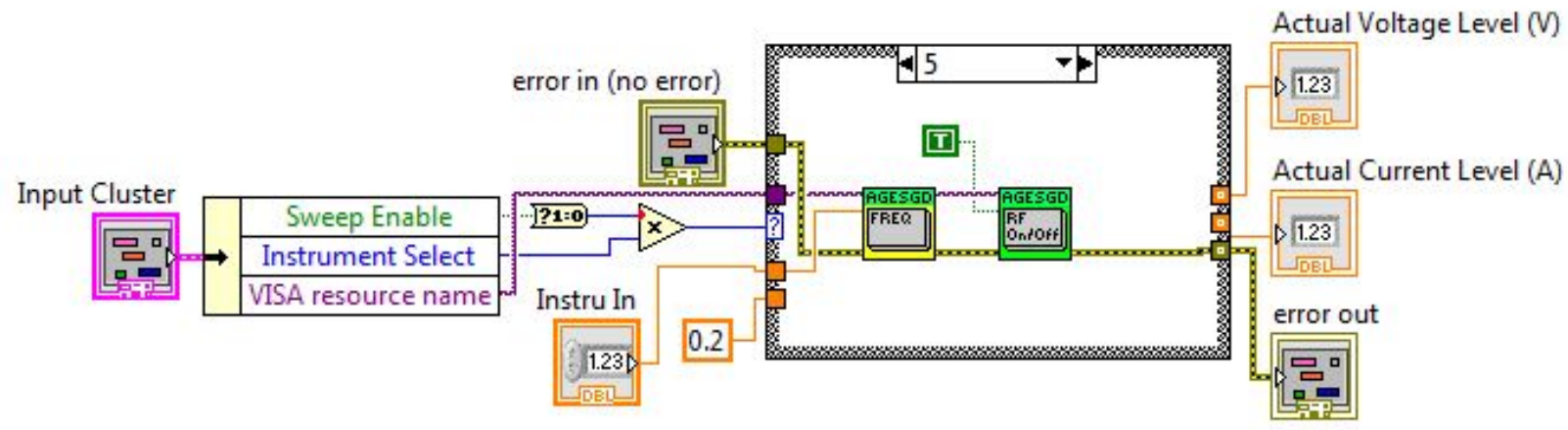

Figure 6 Software block diagram about the setting of the instrument.

\section{Experimental Results}

The proposed test system is verified by measuring the various types of ADCs and the results demonstrate the reliability and convenience of the proposed method. Fig. 7 presents the dynamic measurement results of a sigma-delta ADC [5] and its screen capture. The measured spectra and the dynamic performance data are prominently plotted in real-time. Moreover, the input parameter automated sweep function is applied, and the ADC achieves $84.2 \mathrm{~dB}$ peak SNDR for signal bandwidth of $25 \mathrm{kHz}$ with a sampling clock rate of $10 \mathrm{MHz}$ versus various frequencies and amplitudes of the input signal. The tables in the program front panel detail the dynamic specifications when the measurement is completed, and the tables of corresponding measurement results help the user to fully understand the ADC performance situations with the various input 


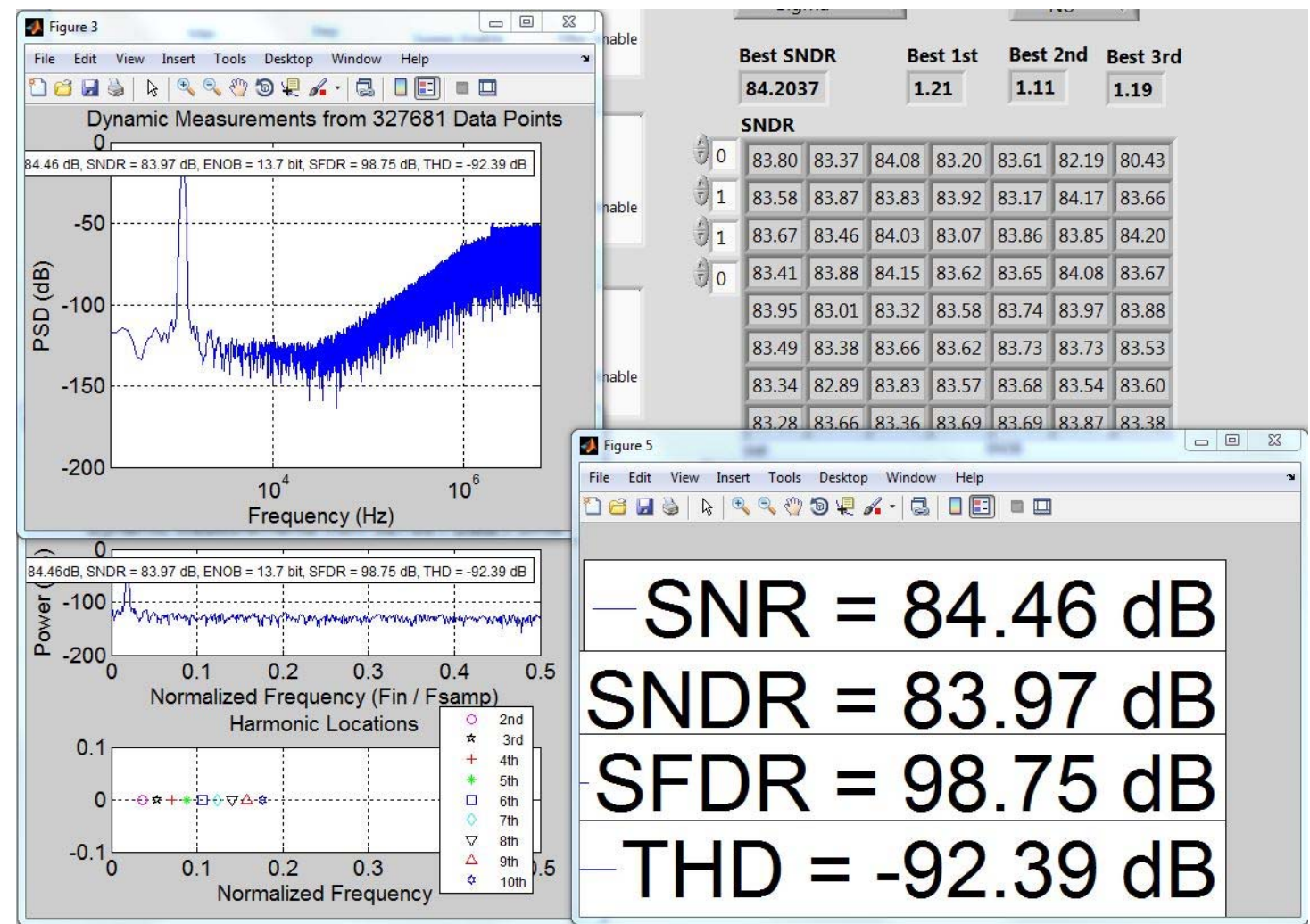

Figure 7 Measurement results of sigma-delta ADC [5].

parameters. Therefore, it benefits to save the user's time and improve the efficiency and precision of the performance data collection.

\section{Conclusions}

This paper presented and demonstrated a well-designed automated test system using various hardware and software components for various types of ADC, considerably improving the quality and efficiency of data converter characterization. Furthermore, the proposed system simplifies ADC measurement through an automated operation. A user-friendly environment allows the selection of numerous input parameters for automatic sweeping reducing test costs and development time.

\section{Acknowledgements}

This work is supported by Macau Science and Technology Development Fund under FDCT/055/2012/A2.

\section{References}

[1] S. Raze, D. Dallet and P. Marchegay, "Industrial Test of A/D Converters in LabVIEW", in IEEE International Workshop on Intelligent Data Acquisition and Advanced Computing Systems: Technology and Applications, pp. 177-180, Sep. 2003.

[2] Ben E. Franklin, Cajetan M. Akujuobi, Warsame Ali, "ADC Automated Testing Using LabVIEW Software," Proceedings of the 2004 American Society for Engineering Education Annual Conference, Sep. 2004.

[3] José Erick de Souza Lima, Carlos A. dos Reis Filho, "Automated Test-Bed for Analog to Digital Converters," in 11th Latin American Test Workshop, pp. 1-5, Mar. 2010. 
[4] Vilmos Pálfi and István Kollár, "Realiable ADC testing using LabVIEW," in 20th IMEKO TC4 International Symposium and 18th International Workshop on ADC Modelling and Testing, Benevento, Italy, Sep. 2014.

[5] Yuan Ren, Sai-Weng Sin, Chi-Seng Lam, Man-Chung Wong, Seng-Pan U, Rui Paulo Martins, "A High DR Multi-Channel Stage-Shared Hybrid Front-End for Integrated Power Electronics Controller," in IEEE Asian Solid-State Circuits Conference, pp. 57-60, Nov. 2016.

[6] “IEEE Standard for Terminology and Test Methods for Analog-to-Digital Converters", IEEE Std 1241-2010, Jan.14,2011. (Revision of IEEE Std. 1241-2000).

[7] M. Burns and G. W. Roberts, An Introduction to Mixed-Signal IC Test and Measurement, New York: Oxford Univ. Press, 2000. 